\title{
Measuring homogeneity of ethno-medicinal knowledge related with different corporeal system: A top down approach
}

\author{
Manish Mathur \\ 18E/564 Chopasni Housing Board, Jodhpur (Rajasthan), INDIA \\ Present Address: Plant Ecology Laboratory, Central Arid Zone Research Institute, Jodhpur (Rajasthan), INDIA \\ E-mail: eco5320@gmail.com \\ Received: September 15, 2014; Revised received: January 24, 2015; Accepted: April 10, 2015
}

\begin{abstract}
In the present study the top down approach has been utilized to quantify homogeneity of ethno-knowledge, frequency of species utilized for a disease related to a specific system as well as identification of corporeal system that are outliers with aim to identify the species and their usefulness for a specific disease that make the system as outlier. The study was carried out for medicinal plants of arid and semiarid region of the Indian Thar area. Three statistical parameters namely, evenness, frequency distribution and regression and residual value analysis were utilized. The study revealed that ethno-medicinal knowledge about 136 species were related with 12 different corporeal systems and frequency distribution classified these 136 species and 12 corporeal systems under 9 different classes. Maximum numbers of taxa are recorded for skeletal, muscle and connective tissues (114) followed by the digestive system (111) and skin and sub-cutanuous system (89). Homogeneity of ethnic- knowledge are restricted for few corporeal systems like respiration, fever, blood and hematopoietic organ, central nervous system, genito-urinary system and circulatory systems, while random and clumped types knowledge distribution were recorded for other corporeal systems. Platykurtic and leptokurtic types of distributions were observed for different corporeal systems. Through use of standard residuals analysis of medico-ethnic-knowledge, digestion system was designated as positive outliers while fever and genito-urinary systems were the negative outliers. Further regression analysis has revealed that with in this region ethnic knowledge about the medicinal properties of a species is largely associated with the regional abundance of taxa.
\end{abstract}

Keywords: Arid and semi arid plants, Ethnic knowledge, Frequency distribution, Residual value analysis, Smith and Wilson Index

\section{INTRODUCTION}

As with all aspects of science, ethno-botany inventories and their analysis must be approached in a logical and systematic fashion. All ethno descriptions and analysis must have a purpose. Nevertheless there are many different approaches are available in mathematics and anthropological sciences that can be adopted and modified for collection, quantification and interpretation of ethno data (quantitative ethnobotany). The collection and analysis of ethno data provide the principal form of different ecosystem services provided to a particular community, habitat or eco-region. However, Majority of these studies are qualitative in nature (Medeiros et al., 2011) and it become very difficult to funnel meaningful information from such binary information's. Further, such information generated data's related with diversity of knowledge that directly or indirectly reflecting the heterogeneity of information's that mislead the process of cultural transmission (Mathur, 2013d). However, little effort has been made to quantify the homogeneity and heterogeneity in combine.

With the availability of modern analytical techniques and the involvement of various branches of sciences in ethno-studies, documentation of various ethno-uses are now supported and interpreted with uses of many statistical techniques (Mathur, 2014b).

In ecological world, species diversity consists of two independent components, richness and evenness. Tuomisto (2012) has defined the evenness as the ratio of diversity/richness. In quantitative ethnobotany different evenness or equitability indices have been used to major the character and distribution of information (homogenization/non-homogenous) of ethnic knowledge (Signorini et al., 2009). Evenness or equitability represents the distribution of individuals among the species. Measures of evenness (or equitability) quantify the unequal representation of species against a hypothetical sample in which all species are equally abundant (Krebs, 1999), i.e., the ratio of the observed diversity to maximum possible diversity. Hence, evenness may be referred to as relative diversity or homogeneity (Brower and Zar, 1977 and Zar, 1984). A low evenness means a high dominance in the use (or presence) of a few species (Begossi, 1996).

When all species are equally abundant, an evenness index would be at a maximum (of 1.0) and decrease towards zero as the relative abundances of the species diverge away from evenness (Ludwig and Reynolds, 
1988). Frequency distribution analysis can provide an insight about the distribution of variables related to a particular system (Williams et al., 2005). This statistical parameters can be evaluated through two sub-parameters like Skewness and Kurtosis and Gamma distribution modeling that can be carried out with Kolmogorov -Simrnov (K-S test). Moerman methods of comparative analysis (Moerman, 1996) can be utilized to quantify homogeneity of ethno-knowledge. The use of this quantitative technique for ethnic-knowledge has been advocated by Amiguet et al. (2005); Bennett and Husby (2008) and Khan et al. (2011). Khan et al. (2011) have utilized this approach to distinguish the relationships between the uses of medicinal plants with their knowledge and with their abundance at a particular eco-region

The objectives of the present study were to evaluate the homogeneity of ethno-knowledge about medicinal plants that are recorded for different diseases related to corporeal systems, quantify the relationships between the ethnicknowledge and the regional availability of a species and identification of corporeal system that are outliers with aim to identify the species and their usefulness for a specific disease that make the system as outlier.

\section{MATERIALS AND METHODS}

In the present study the three statistical parameters namely, evenness, frequency distribution and regression and residual value analysis were utilized. Database pertaining to the ethno-medicinal uses of 136 arid and semi arid species was prepared with the help of author previous works Mathur (2005), Mathur (2012a and b), Mathur and Sundaramoorthy (2013), Mathur (2013a, b and c) and Kumar et al. (2014). These previous studies had reported the relative importance value of different arid and semi arid plants, their sensitivity index, their economic assessment and conservation priorities, different ecosystem services (cultural, provisional and regulatory) provided by them, quantification of the types and erosion of cultural transmission, medicinal plants primarily useful for various diseases related to different corporeal system like central nervous, gastro-intestinal, reproductive systems and the market analysis of certain plants in relation to their medicinal properties.

Evenness: Evenness based on Shannon and Weaver and Simpson indexes were quantified according to Payne et al., 2005). While Simpson's index of diversity, measures the probability that two individuals selected at random from a sample will belong to same species were quantified according to Mathur (2014a).

Simpson' sIndex of Diversity $=\left\{\begin{array}{c}\text { Probability of picking two } \\ \text { organism at random } \\ \text { that are different species }\end{array}\right\}=1-\left\{\begin{array}{c}\text { Probability of pickng two } \\ \text { orgamism that are the } \\ \text { same species }\end{array}\right\}$

Thus

$$
1-D=1-\Sigma(P i)^{2}
$$

Where: $(1-\mathrm{D})=$ Simpson's Index of Diversity and $\mathrm{Pi}=$ proportion of individuals of species I in the community. However, Smith and Wilson's Index, which is based on variance in the abundance of the species (Mathur, 2014b) and in this method the variance was measured over the log of the abundance in order to use proportional differences rather than absolute differences in abundance.

$$
E_{\text {Var }}=1.0-\left(\frac{2}{\pi}\right)\left[\operatorname{arctab}\left\{\frac{\sum_{i=1}^{S}\left(\log e\left(n_{i}\right)-\sum_{j=1}^{S} \log _{\theta}\left(n_{j}\right) / S\right)^{2}}{S}\right\}\right]
$$

Where the arctangent is measured as an angle in radians and $\mathrm{E}_{\mathrm{var}}=$ Smith and Wilson's index of evenness, $\mathrm{n}_{\mathrm{i}}=$ number of individuals in species I in sample, $\mathrm{n}_{\mathrm{j}}=$ number of individual in species $\mathrm{j}$ in sample, $\mathrm{S}=$ Number of species in entire sample. The arctangent can be calculated by simple ATAN formula provided in excel spreadsheets. Value of $<1$ indicates the clumped, 1 for uniform and $>1$ for random pattern.

\section{Frequency distribution}

To quantify the use of a different species for a disease related to a particular corporeal system, frequency distribution analysis was carried out with parameters, namely skewness and kurtosis and the Kolmogorov Smirnov test. Skewness indicates the scatter patterns of the information/knowledge and their distance from the original standard mean. Skewness also indicted the direction (asymmetrical) i.e. toward positive or negative skewness. For no skewness $($ mean $=$ median $=$ mode $)$, positive skewness (mean $>$ median $>$ mode) and mean $<$ median $<$ mode for negative skewness. Kurtosis is utilized to study the nature of peak of frequency distribution. This parameter provides the concentration of frequencies in the middle of the distribution. On the basis of nature of the peak, frequency distribution graph may be mesokurtic (normal), leptokurtic (dense at middle portion) and platykurtic (indicate the lesser frequencies at central). In a normal distribution the value of skewness and kurtosis are equal or approximate zero.

Gamma distribution was performed to simulate the distribution patterns of ethno-information among multiple informants. The gamma distribution is described by the probability density function. It's a non parametric test for the equality of continuous, a one-dimensional probability distribution that can be used to compare a sample with a reference probability distribution. The Kolmogorov Smirnov test allows to test if the biggest difference between the empirical and theoretical cumulative distribution functions is above a critical value or not. This test is used to decide if a sample comes from a hypothesized continuous distribution. It is based on the empirical cumulative distribution function (ECDF).

The Kolmogorov-Smirnov statistic (D) is based on the largest vertical difference between the theoretical and the empirical cumulative distribution function: 
Further for this test the null and the alternative hypotheses are as follows $\left(H_{0}\right)$ : the data follow the normal distribution and $\left(H_{A}\right)$ : the data do not follow the normal distribution.

\section{Residual value analysis}

Moerman (1996) had developed a linear regression model including residual analysis which assessed the importance of a family as a source of medicines or food used on the overall proportion of correspondingly used species in the flora. The regression analysis seeks to quantify the degree to which a family has an unexpectedly high or low number of medicinal species relative to the overall proportion of medicinal species in the flora i.e. averaged by the least squares regression lines. Regression analysis was performed with the help of curve expert software. Numbers of total medicinal properties were plotted against the number of taxa in each corporeal system. Outliers were calculated for each corporeal system. A regression outlier is an observation that has an unusual value of the dependent variable Y, Conditional on its value of the independent variable X. Outliers (standardized residuals and Cook distance) plays an important role in the regression, and both can affect the regression model. In the present investigation standardized residuals was quantified as per suggested by Wilcox (2001).

\section{RESULTS AND DISCUSSION}

In this study, medico-ethno-knowledge of 136 plant species related with different corporeal systems have been evaluated for their knowledge homogeneity (Table 1.). Species that claimed maximum for disease related with each corporeal system are presented in table 1 and for example Achyranthies aspera, Adhatoda vasica, Boswellia serrata, Calotropis procera and Ocimum sanctum were recorded for five different diseases related with respiration system, similarly Tamarindus indica had been claimed for eight different disease related with digestion system and Azadirachta indica had been reported maximum time (nine) for their medicinal properties against skin and sub-cutanuous system. Under each corporeal system, the number of taxa claimed for their medicinal uses and the total number of uses of each species for a specific disease with relation to their respective corporeal system are presented in table 2 . Maximum numbers of taxa are recorded for skeletal, muscle and connective tissues (114) followed by the digestive system (111) and skin and sub-cutanuous system (89).

Yang and Gao (2011) have advocated that calculation of the evenness value helps to find out whether the number of species utilized among the tribes/groups are high or low. A low evenness means a high dominance with the use of the few species, while value approaches to 1 indicates the homogenous distribution of knowledge and use. In this study diversity based ( $\left.H^{\prime}\right)$ and $(\lambda)$ evenness indices revealed the homogenous distribution of ethnic-knowledge amongst the arid and semi arid communities. The values of both these indices recorded $(\mathrm{P}>0.5)$ and approached toward one that indicates homogeneity. However Smith and Wilson index of evenness showed a different scenario. This index revealed the uniformity (as the value of this index approached toward one) of ethno-knowledge for disease related to corporeal systems like respiration, fever, blood and hematopoietic organ, central nervous system, genitor-urinary system and circulatory systems (Table 2).

The index value to corporeal systems like digestion, reproduction, skin and sub-cutanuous and skeletal, muscle and connective tissues were recorded more than one (1.11 to 1.27$)$ that indicates the random pattern of ethnic-knowledge. For circulatory (0.90) and endocrine gland, metabolism and nutrition systems (0.89), the index value was recorded less than one indicated the clumped pattern of knowledge or in other words the medicinal knowledge about disease related with these two corporeal system are prevailing within certain groups only. Such information's are very useful to quantify the transmission process of ethnic-knowledge and factors affecting them. The departure of ethnic- knowledge from homogeneity can be explained by the factors associated with technology advancement, fit between new and previous knowledge, external representation of knowledge (Leonti, 2011), social network structure and social institutions (Tehrani and Collard, 2009 and Henrich and Henrich, 2010), or inter-group conflict (Di Cosmo, 2002) as well as copying and cultural drift that can also drive changes in cultural knowledge (Bentley et al., 2004; Mathur 2013d and 2014b).

In the absence of any suppression factors for transmission of ethnic-knowledge, the random pattern of knowledge can be positively considered as viable sources of information that may expand in all possible directions. Thus temporal surveillance for ethnic knowledge related with disease of digestion, reproduction, skin and sub-cutanuous and skeletal, muscle and connective tissues corporeal systems are essential. However, for a clumped pattern of ethnic -knowledge, there are certain factors need to be identified involving, impact of local abundance of plant species most useful for systems like circulatory and endocrine gland, metabolism and nutrition systems, identification of factors affecting the transmission of such knowledge (Mathur, 2013d).

Results of frequency distribution are presented in tables 3 and 4 . Frequency distribution categorized the 12 corporeal systems and 136 species in to 9 different classes (Table 3). Data revealed (Table 4) that for nine different corporeal systems (except respiration, digestion and skeletal, muscle and connective tissues) skewness were recorded more than one and this indicated the positive skewness (mean $>$ median $>$ mode), that means the distribution of ethnic-knowledge about each species for different diseases related with a particular corporeal system has extreme scores above the mean that are a typical of the majority of scores. Kurtosis describes the 
Table 1. Species name and their various uses against the different ailments related with 12 corporal systems.

\begin{tabular}{|c|c|c|c|c|c|c|c|c|c|c|c|c|}
\hline \multirow[b]{2}{*}{ Species name } & \multicolumn{12}{|c|}{ Name of corporeal systems } \\
\hline & 1 & 2 & 3 & 4 & 5 & 6 & 7 & 8 & 9 & 10 & 11 & 12 \\
\hline Abrus precatorius $\mathrm{L}$ & 2 & 2 & 3 & 1 & 0 & 1 & 1 & 0 & 0 & 0 & 4 & 3 \\
\hline Abutilon indicum (L.) Sweet & 1 & 5 & 3 & 2 & 0 & 2 & 1 & 0 & 1 & 0 & 1 & 2 \\
\hline Acacia nilotica $\mathrm{L}$. & 2 & 1 & 0 & 0 & 0 & 0 & 1 & 0 & 1 & 0 & 4 & 4 \\
\hline Acacia senegal Willd. & 2 & 1 & 0 & 1 & 0 & 0 & 0 & 0 & 1 & 0 & 2 & 2 \\
\hline Achyranthies aspera L. & 5 & 6 & 3 & 0 & 1 & 3 & 2 & 0 & 1 & 0 & 3 & 4 \\
\hline Adhatoda vasica Nees & 5 & 5 & 0 & 0 & 0 & 1 & 0 & 0 & 0 & 0 & 1 & 2 \\
\hline Aegle marmelos Correa ex Koen. & 2 & 6 & 0 & 2 & 1 & 1 & 0 & 0 & 1 & 0 & 1 & 1 \\
\hline Albizia lebbeck Benth. & 0 & 1 & 0 & 0 & 0 & 2 & 0 & 0 & 2 & 0 & 5 & 2 \\
\hline Aloe vera Mill. & 1 & 6 & 2 & 1 & 1 & 1 & 1 & 0 & 2 & 1 & 2 & 6 \\
\hline Amaranthus spinosus L. & 1 & 3 & 1 & 1 & 1 & 1 & 1 & 0 & 0 & 0 & 3 & 3 \\
\hline Andrographis paniculata Wall. ex Nees & 1 & 2 & 1 & 2 & 1 & 1 & 0 & 0 & 0 & 1 & 0 & 1 \\
\hline Anogeissus pendula Edgew. & 0 & 2 & 0 & 0 & 1 & 0 & 1 & 0 & 1 & 0 & 2 & 3 \\
\hline Argemone mexicana L. & 4 & 3 & 0 & 2 & 0 & 0 & 0 & 0 & 3 & 0 & 4 & 3 \\
\hline Argyreia nervosa (Brum. f.) Boj. & 0 & 0 & 1 & 0 & 1 & 0 & 1 & 0 & 0 & 0 & 1 & 2 \\
\hline Aristolochia bracteata Retz. & 0 & 4 & 1 & 1 & 0 & 0 & 0 & 0 & 0 & 0 & 2 & 2 \\
\hline Artemisia annua $\mathrm{L}$. & 1 & 0 & 0 & 2 & 0 & 0 & 0 & 0 & 0 & 0 & 0 & 0 \\
\hline Asparagus racemosus Willd. & 4 & 4 & 4 & 0 & 0 & 2 & 2 & 1 & 1 & 0 & 0 & 4 \\
\hline Azadirachta indica A. Juss. & 2 & 4 & 0 & 4 & 1 & 0 & 1 & 0 & 4 & 0 & 9 & 5 \\
\hline Balanites aegyptiaca (L.) Delile & 1 & 4 & 2 & 0 & 0 & 0 & 0 & 0 & 0 & 0 & 4 & 1 \\
\hline Barleria acanthoides Vahl. & 0 & 0 & 0 & 0 & 0 & 0 & 0 & 0 & 0 & 0 & 0 & 2 \\
\hline Barleria prionitis L. & 4 & 0 & 0 & 1 & 0 & 0 & 0 & 0 & 0 & 0 & 2 & 5 \\
\hline Blepharis edulis Forssk. & 0 & 0 & 1 & 0 & 0 & 0 & 0 & 0 & 0 & 0 & 0 & 1 \\
\hline Blepharis sindica Stocks ex T. Anders & 2 & 0 & 2 & 0 & 1 & 0 & 1 & 0 & 1 & 1 & 1 & 1 \\
\hline Boerhavia diffusa L. & 1 & 5 & 1 & 3 & 1 & 2 & 2 & 1 & 2 & 0 & 0 & 4 \\
\hline Boswellia serrata Roxb. & 5 & 5 & 0 & 0 & 0 & 0 & 1 & 0 & 0 & 0 & 2 & 5 \\
\hline Butea monosperma (Lam.) Kuntze & 1 & 3 & 2 & 1 & 1 & 0 & 1 & 0 & 0 & 0 & 2 & 3 \\
\hline Calligonum polygonoides L. & 1 & 1 & 0 & 0 & 0 & 0 & 0 & 0 & 1 & 0 & 0 & 1 \\
\hline Calotropis procera (Ait.) R. Br. & 5 & 3 & 0 & 1 & 0 & 2 & 0 & 0 & 1 & 0 & 0 & 4 \\
\hline Capparis decidua Edgew. & 2 & 0 & 0 & 1 & 0 & 0 & 0 & 1 & 0 & 0 & 1 & 4 \\
\hline Cardiospermum halicacabum L. & 0 & 2 & 1 & 0 & 0 & 3 & 1 & 0 & 1 & 0 & 2 & 2 \\
\hline Cassia angustifolia Vahl & 1 & 5 & 0 & 0 & 0 & 0 & 0 & 0 & 0 & 0 & 3 & 2 \\
\hline Cassia fistula L. & 1 & 4 & 0 & 3 & 1 & 1 & 0 & 0 & 0 & 0 & 4 & 3 \\
\hline Cassia occidentalis L. & 0 & 3 & 1 & 1 & 0 & 0 & 1 & 0 & 0 & 0 & 4 & 0 \\
\hline Catharanthus roseus G. Don & 2 & 1 & 1 & 0 & 3 & 1 & 2 & 0 & 0 & 0 & 0 & 0 \\
\hline Cissus quadrangularis L. & 0 & 6 & 1 & 0 & 1 & 1 & 0 & 0 & 0 & 0 & 2 & 1 \\
\hline Citrullus colocynthis Schrad. & 0 & 4 & 0 & 2 & 0 & 1 & 1 & 0 & 1 & 0 & 0 & 2 \\
\hline Citrullus lanatus Thunb. & 0 & 1 & 1 & 0 & 0 & 1 & 1 & 0 & 0 & 0 & 0 & 1 \\
\hline Chloris virgata $\mathrm{Sw}$. & 0 & 5 & 0 & 1 & 0 & 0 & 1 & 1 & 1 & 1 & 0 & 2 \\
\hline Clerodendrum phlomoides L.f. & 0 & 5 & 0 & 1 & 0 & 0 & 1 & 1 & 0 & 0 & 1 & 2 \\
\hline
\end{tabular}


Table 1. Contd.

\begin{tabular}{|c|c|c|c|c|c|c|c|c|c|c|c|c|}
\hline Cocculus hirsutus (L.) Diels & 0 & 3 & 2 & 1 & 1 & 0 & 1 & 0 & 1 & 0 & 1 & 2 \\
\hline Cocculus pendulus (Forsk.) Diels & 0 & 0 & 0 & 1 & 1 & 1 & 1 & 0 & 0 & 0 & 1 & 1 \\
\hline Commiphora wightii (Arn.) Bhandari & 4 & 1 & 0 & 0 & 4 & 0 & 1 & 0 & 0 & 1 & 3 & 3 \\
\hline Convolvulus microphyllus Sieb. Ex Spreng & 0 & 1 & 0 & 0 & 0 & 1 & 0 & 0 & 0 & 0 & 0 & 1 \\
\hline Corchorus depressus (L.) C. Chr. & 0 & 1 & 3 & 0 & 1 & 0 & 0 & 0 & 0 & 1 & 0 & 1 \\
\hline Crotalaria burhia Buch.-Ham & 3 & 0 & 0 & 0 & 0 & 1 & 1 & 0 & 0 & 0 & 0 & 3 \\
\hline Cucumis callosus (Rottl.) Cogn. & 0 & 2 & 0 & 0 & 0 & 0 & 0 & 0 & 0 & 0 & 0 & 1 \\
\hline Cynodon dactylon Pers. & 1 & 3 & 0 & 0 & 0 & 0 & 2 & 0 & 3 & 0 & 2 & 3 \\
\hline Cyperus rotundus $\mathrm{L}$. & 0 & 5 & 1 & 0 & 0 & 0 & 1 & 0 & 1 & 0 & 1 & 2 \\
\hline Celosia argente $\mathrm{L}$. & 0 & 0 & 2 & 0 & 1 & 0 & 0 & 0 & 1 & 0 & 0 & 0 \\
\hline Chenopodium album $\mathrm{L}$. & 0 & 5 & 2 & 0 & 2 & 0 & 1 & 1 & 1 & 0 & 1 & 1 \\
\hline Cressa cretica $\mathrm{L}$. & 3 & 5 & 1 & 0 & 3 & 0 & 1 & 0 & 0 & 0 & 1 & 3 \\
\hline Cyperus scariosus $\mathrm{R}$. Br. & 0 & 1 & 1 & 0 & 0 & 1 & 1 & 1 & 0 & 0 & 0 & 1 \\
\hline Chloris virgata $\mathrm{Sw}$. & 2 & 0 & 1 & 0 & 0 & 0 & 1 & 0 & 0 & 0 & 0 & 0 \\
\hline Cordia myxa L. & 2 & 2 & 2 & 1 & 0 & 0 & 0 & 0 & 0 & 0 & 1 & 1 \\
\hline Cymbopogon citratus Stapf & 1 & 4 & 1 & 0 & 0 & 0 & 0 & 0 & 0 & 0 & 2 & 1 \\
\hline Corchorus trilocularis L. & 0 & 2 & 1 & 1 & 0 & 1 & 0 & 0 & 0 & 0 & 2 & 3 \\
\hline Datura metel L. & 3 & 1 & 1 & 1 & 0 & 0 & 0 & 2 & 1 & 0 & 4 & 5 \\
\hline Desmostachya bipinnata Stapf & 1 & 1 & 1 & 1 & 0 & 0 & 1 & 0 & 0 & 0 & 1 & 2 \\
\hline Dalbergia sissoo Roxb & 1 & 8 & 3 & 1 & 0 & 1 & 2 & 0 & 1 & 0 & 5 & 5 \\
\hline Echinops echinatus Roxb. & 2 & 1 & 2 & 0 & 0 & 1 & 1 & 0 & 1 & 0 & 1 & 1 \\
\hline Eclipta prostrata (L.) L. & 3 & 3 & 1 & 1 & 1 & 1 & 0 & 0 & 0 & 1 & 3 & 2 \\
\hline Emblica officinalis Gaertn & 2 & 4 & 0 & 0 & 3 & 0 & 1 & 1 & 1 & 0 & 0 & 5 \\
\hline Ephedra foliata Boiss. \& Kotschy & 3 & 0 & 0 & 0 & 0 & 0 & 0 & 1 & 0 & 0 & 0 & 1 \\
\hline Euphorbia caducifolia Haines. & 2 & 2 & 1 & 1 & 1 & 1 & 0 & 0 & 0 & 0 & 3 & 2 \\
\hline Euphorbia hirta $\mathrm{L}$. & 3 & 1 & 1 & 0 & 0 & 0 & 0 & 0 & 0 & 0 & 3 & 1 \\
\hline Evovulus alsinoides $\mathrm{L}$. & 2 & 2 & 2 & 1 & 0 & 1 & 0 & 0 & 0 & 0 & 2 & 2 \\
\hline Fagonia indica Burm. f. & 1 & 2 & 0 & 1 & 1 & 0 & 0 & 0 & 0 & 0 & 2 & 0 \\
\hline Ficus religiosa $\mathrm{L}$. & 2 & 5 & 1 & 1 & 2 & 0 & 0 & 1 & 0 & 0 & 2 & 0 \\
\hline Ficus bengalensis L. & 0 & 1 & 0 & 0 & 1 & 0 & 0 & 0 & 0 & 0 & 2 & 6 \\
\hline Fumaria indica Pugsley & 0 & 1 & 0 & 2 & 1 & 0 & 1 & 0 & 1 & 1 & 1 & 0 \\
\hline Glycyrrhiza glabra L. & 3 & 2 & 0 & 0 & 1 & 0 & 1 & 0 & 0 & 1 & 3 & 1 \\
\hline Grewia tenax (Forsk.) Aschers. \& Schwf & 3 & 1 & 0 & 0 & 0 & 0 & 0 & 0 & 0 & 0 & 1 & 0 \\
\hline Grewia tiliaefolia Vahl & 4 & 2 & 1 & 1 & 2 & 0 & 0 & 0 & 0 & 0 & 3 & 4 \\
\hline Grewia populifolia Vahl & 0 & 1 & 2 & 0 & 0 & 0 & 0 & 0 & 0 & 0 & 0 & 0 \\
\hline Heliotropium indicum $\mathrm{L}$. & 1 & 1 & 2 & 1 & 0 & 0 & 1 & 0 & 0 & 0 & 2 & 2 \\
\hline Hibiscus rosa-sinensis $\mathrm{L}$. & 1 & 1 & 5 & 1 & 0 & 0 & 1 & 2 & 0 & 0 & 2 & 4 \\
\hline Holarrhena antidysenterica (L.) Wall. & 4 & 4 & 1 & 0 & 0 & 1 & 0 & 0 & 0 & 1 & 1 & 1 \\
\hline Indigofera linnaei Ali. & 0 & 0 & 1 & 0 & 0 & 2 & 1 & 0 & 0 & 0 & 0 & 1 \\
\hline Jatropha curcas $\mathrm{L}$. & 0 & 1 & 0 & 0 & 2 & 0 & 0 & 0 & 0 & 0 & 4 & 5 \\
\hline Lawsonia inermis L. & 1 & 0 & 0 & 1 & 0 & 0 & 0 & 0 & 1 & 0 & 4 & 2 \\
\hline Leptadenia reticulata Wight \& Arn. & 0 & 1 & 0 & 0 & 0 & 0 & 0 & 0 & 0 & 0 & 0 & 1 \\
\hline
\end{tabular}


Table 1. Contd.

Lycium barbarum L.

Lycium chinense Mill.

Lantana camara L.

Leucas aspera Spreng.

Majorana hortensis Moench. (Ruiz \& Pav.) Loes

Maytenus emarginata ( Ruiz \& Pav.) Loes

Mimosa pudica L.

Mimosa hamata Willd.

Mollugo cerviana Ser.

Moringa oleifera Lam.

Mucuna pruriens Baker.

Murraya koenigii (L.) Spreng

Neurada procumbens L.

Nyctanthes arbor-tristris L.

Ocimum sanctum L.

Opuntia elatior (Mill.) Willd.

Pedalium murex L.

Peganum harmala L.

Pergularia daemia (Forsk.) Chiov.

Phyllanthus amarus Schum.

Phyllanthus fraternus Webst.

Plantago ovata Forsk.

Polygala senega L.

Portulaca oleracea L.

Prosopis cineraria Druce

Pulicaria crispa Oliver

Punica granatum $\mathrm{L}$.

Ricinus communis L.

Salvadora oleoides Decne

Salvadora persica L.

Sarcostemma acidum Voigt

Sida cordifolia L.

Sisymbrium irio L.

Solanum nigrum L.

Solanum surattense Burm. f.

Sphaeranthus indicus L.

Saccharum spontaneum L.

Solanum xanthcarpum Schrad. \& Wendl

Tamarindus indica $\mathrm{L}$.

Tecomella undulata (Sm) Seem.

$\begin{array}{lllllllllll}2 & 0 & 2 & 0 & 0 & 0 & 1 & 0 & 0 & 0 & 0 \\ 0 & 0 & 2 & 0 & 2 & 0 & 0 & 0 & 0 & 0 & 0 \\ 0 & 2 & 0 & 1 & 0 & 0 & 0 & 0 & 0 & 0 & 0 \\ 1 & 3 & 0 & 1 & 0 & 0 & 0 & 0 & 0 & 0 & 1 \\ 3 & 1 & 0 & 0 & 1 & 0 & 1 & 0 & 0 & 0 & 0\end{array}$

$\begin{array}{llllllllllll}0 & 1 & 0 & 1 & 3 & 0 & 0 & 0 & 0 & 1 & 0 & 2\end{array}$

$\begin{array}{llllllllllll}1 & 3 & 0 & 0 & 0 & 0 & 1 & 0 & 0 & 0 & 2 & 0\end{array}$

$\begin{array}{llllllllllll}1 & 2 & 3 & 1 & 2 & 0 & 1 & 0 & 0 & 0 & 7 & 3\end{array}$

$\begin{array}{llllllllllll}0 & 1 & 0 & 0 & 2 & 0 & 0 & 0 & 0 & 0 & 1 & 1\end{array}$

$\begin{array}{llllllllllll}1 & 1 & 1 & 0 & 1 & 3 & 1 & 1 & 0 & 1 & 0 & 6\end{array}$

$\begin{array}{llllllllllll}0 & 2 & 1 & 0 & 0 & 2 & 1 & 1 & 0 & 0 & 1 & 2\end{array}$

$\begin{array}{llllllllllll}0 & 4 & 0 & 0 & 1 & 2 & 1 & 0 & 1 & 0 & 2 & 0\end{array}$

$\begin{array}{llllllllllll}0 & 0 & 1 & 0 & 0 & 0 & 0 & 1 & 0 & 0 & 0 & 1\end{array}$

$\begin{array}{llllllllllll}2 & 0 & 0 & 2 & 0 & 1 & 0 & 0 & 0 & 0 & 3 & 3\end{array}$

$\begin{array}{llllllllllll}5 & 5 & 0 & 2 & 0 & 3 & 1 & 0 & 2 & 0 & 2 & 3\end{array}$

$\begin{array}{llllllllllll}1 & 1 & 1 & 0 & 0 & 0 & 0 & 0 & 1 & 0 & 0 & 1\end{array}$

$\begin{array}{llllllllllll}0 & 2 & 4 & 0 & 0 & 1 & 2 & 0 & 0 & 0 & 0 & 0\end{array}$

$\begin{array}{llllllllllll}1 & 3 & 1 & 1 & 0 & 2 & 0 & 0 & 0 & 0 & 2 & 2\end{array}$

$\begin{array}{llllllllllll}2 & 3 & 2 & 0 & 0 & 0 & 0 & 0 & 0 & 0 & 1 & 1\end{array}$

$\begin{array}{llllllllllll}0 & 0 & 1 & 1 & 0 & 0 & 0 & 0 & 0 & 0 & 2 & 0\end{array}$

$\begin{array}{llllllllllll}0 & 2 & 0 & 3 & 0 & 0 & 2 & 0 & 0 & 0 & 3 & 2\end{array}$

$\begin{array}{llllllllllll}0 & 5 & 2 & 0 & 0 & 0 & 0 & 0 & 0 & 0 & 0 & 1\end{array}$

$\begin{array}{llllllllllll}3 & 1 & 0 & 0 & 0 & 0 & 0 & 0 & 0 & 0 & 0 & 0\end{array}$

$\begin{array}{llllllllllll}0 & 2 & 0 & 0 & 2 & 0 & 1 & 0 & 0 & 1 & 2 & 1\end{array}$

$\begin{array}{llllllllllll}0 & 1 & 1 & 0 & 0 & 1 & 0 & 0 & 0 & 0 & 0 & 2\end{array}$

$\begin{array}{llllllllllll}3 & 0 & 0 & 2 & 0 & 0 & 0 & 0 & 0 & 0 & 1 & 0\end{array}$

$\begin{array}{llllllllllll}4 & 6 & 1 & 0 & 0 & 0 & 0 & 0 & 0 & 0 & 0 & 2\end{array}$

$\begin{array}{llllllllllll}0 & 3 & 1 & 0 & 1 & 1 & 0 & 0 & 2 & 1 & 4 & 1\end{array}$

$\begin{array}{llllllllllll}2 & 0 & 0 & 0 & 0 & 0 & 0 & 0 & 0 & 0 & 0 & 3\end{array}$

$\begin{array}{llllllllllll}2 & 4 & 1 & 0 & 0 & 1 & 1 & 0 & 0 & 0 & 1 & 3\end{array}$

$\begin{array}{llllllllllll}0 & 1 & 0 & 0 & 0 & 0 & 0 & 0 & 0 & 0 & 0 & 1\end{array}$

$\begin{array}{llllllllllll}2 & 3 & 1 & 1 & 1 & 1 & 1 & 0 & 0 & 0 & 1 & 1\end{array}$

$\begin{array}{llllllllllll}2 & 0 & 0 & 1 & 0 & 0 & 0 & 0 & 0 & 0 & 0 & 0\end{array}$

$\begin{array}{llllllllllll}2 & 5 & 1 & 1 & 0 & 0 & 1 & 0 & 0 & 2 & 6 & 3\end{array}$

$\begin{array}{llllllllllll}4 & 4 & 0 & 0 & 1 & 1 & 1 & 0 & 0 & 1 & 1 & 3\end{array}$

$\begin{array}{llllllllllll}1 & 6 & 2 & 1 & 1 & 0 & 0 & 3 & 2 & 0 & 2 & 0\end{array}$

$\begin{array}{llllllllllll}0 & 2 & 1 & 1 & 2 & 1 & 1 & 0 & 0 & 0 & 0 & 2\end{array}$

$\begin{array}{llllllllllll}2 & 0 & 1 & 0 & 2 & 0 & 2 & 0 & 0 & 1 & 0 & 3\end{array}$

$\begin{array}{llllllllllll}1 & 8 & 0 & 3 & 0 & 0 & 0 & 0 & 1 & 0 & 0 & 3\end{array}$

$\begin{array}{llllllllllll}1 & 0 & 0 & 1 & 1 & 0 & 0 & 0 & 0 & 0 & 3 & 1\end{array}$ 
Table 1. Contd.

\begin{tabular}{lllllllllllll}
\hline Tephrosia purpurea (L.) Pers. & 2 & 6 & 0 & 0 & 0 & 0 & 2 & 0 & 0 & 1 & 2 & 3 \\
Terminalia arjuna (Roxb) Wt \& Arn. & 3 & 2 & 1 & 0 & 4 & 0 & 1 & 1 & 0 & 1 & 3 & 4 \\
Tinospora cordifolia (Willd.) Miers ex & 0 & 3 & 0 & 1 & 1 & 1 & 1 & 0 & 1 & 1 & 0 & 3 \\
Hook. f. \& Thoms. & & & & & & & & & & & \\
Trianthema portulacastrum L. & 0 & 3 & 0 & 1 & 0 & 1 & 2 & 0 & 1 & 1 & 1 & 1 \\
Tribulus terrestris L. & 0 & 3 & 2 & 0 & 0 & 0 & 3 & 0 & 0 & 0 & 0 & 4 \\
Trigonella foenum-graecum L. & 1 & 4 & 0 & 2 & 1 & 0 & 0 & 0 & 0 & 0 & 1 & 6 \\
Tylophora indica (Burm. f.) Merrill & 3 & 3 & 0 & 0 & 0 & 0 & 0 & 0 & 0 & 0 & 1 & 1 \\
Urginea indica Kunth. & 3 & 1 & 0 & 0 & 0 & 0 & 0 & 1 & 0 & 0 & 4 & 4 \\
Vernonia cinerea Less. & 1 & 5 & 0 & 2 & 1 & 0 & 1 & 0 & 2 & 0 & 5 & 1 \\
Vitex negundo L. & 2 & 2 & 0 & 2 & 0 & 2 & 1 & 0 & 0 & 1 & 1 & 6 \\
Vetiveria zizanoides (L.) Nash & 1 & 3 & 0 & 1 & 1 & 1 & 1 & 1 & 0 & 0 & 1 & 2 \\
Withania somifera Dunal & 3 & 1 & 1 & 1 & 1 & 3 & 1 & 0 & 0 & 0 & 1 & 3 \\
Ziziphus mauritiana Lam. & 4 & 5 & 1 & 1 & 1 & 0 & 0 & 0 & 0 & 0 & 0 & 3 \\
Ziziphus nummularia (Burm. f.) Wight \& Arn. & 2 & 0 & 1 & 1 & 0 & 0 & 0 & 0 & 0 & 0 & 3 & 0 \\
Zygophyllum simplex L. & 0 & 1 & 0 & 0 & 0 & 0 & 0 & 0 & 3 & 0 & 0 & 0 \\
\hline Maximum & $\mathbf{5}$ & $\mathbf{8}$ & $\mathbf{5}$ & $\mathbf{4}$ & $\mathbf{4}$ & $\mathbf{3}$ & $\mathbf{3}$ & $\mathbf{3}$ & $\mathbf{4}$ & $\mathbf{2}$ & $\mathbf{9}$ & $\mathbf{6}$ \\
\hline
\end{tabular}

1. Respiration, 2. Digestion, 3. Reproduction, 4. Fever, 5. Blood and Hematopoietic Organ, 6. Central Nervous System, 7. Genito-Urinary System, 8. Circulatory System, 9. Sensory System, 10. Endocrine Gland, Metabolism and Nutrition, 11. Skin and Sub-Cutanuous System and 12. Skeletal, Muscle and Connective Tissues

Table 2. Evenness indices for different corporeal systems.

\begin{tabular}{|c|c|c|c|c|c|}
\hline \multirow[t]{2}{*}{ Different corporeal systems } & \multicolumn{2}{|c|}{ Taxonomic information } & \multicolumn{3}{|c|}{ Evenness based indexes } \\
\hline & Taxa & Individuals & Simpson & $\begin{array}{c}\text { Shannon and } \\
\text { Weaver }\end{array}$ & $\begin{array}{l}\text { Smith and } \\
\text { Wilson }\end{array}$ \\
\hline Respiration & 87 & 191 & 0.77 & 0.96 & 0.98 \\
\hline Digestion & 111 & 322 & 0.73 & 0.95 & 1.21 \\
\hline Reproduction & 71 & 111 & 0.74 & 0.96 & 1.11 \\
\hline Fever & 64 & 88 & 0.80 & 0.97 & 0.97 \\
\hline Blood and hematopoietic organ & 53 & 77 & 0.78 & 0.963 & 0.96 \\
\hline Central nervous system & 48 & 68 & 0.81 & 0.97 & 0.96 \\
\hline Genito-urinary system & 66 & 79 & 0.88 & 0.98 & 0.96 \\
\hline Circulatory system & 19 & 23 & 0.84 & 0.97 & 0.90 \\
\hline Sensory system & 39 & 55 & 0.78 & 0.96 & 0.95 \\
\hline $\begin{array}{l}\text { Endocrine gland, metabolism and } \\
\text { nutrition }\end{array}$ & 22 & 23 & 0.96 & 0.99 & 0.89 \\
\hline Skin and sub-cutanuous system & 89 & 207 & 0.70 & 0.96 & 1.27 \\
\hline $\begin{array}{l}\text { Skeletal, muscle and connective } \\
\text { tissues }\end{array}$ & 114 & 280 & 0.75 & 0.95 & 1.24 \\
\hline
\end{tabular}

nature of the frequency distribution peak.

In present study the graphical representation of observed and theoretical frequencies are presented in Fig.1. Platykurtic type distribution represented negative values compare to normal kurtosis and these were recorded for corporeal systems like respiration (Kurtosis value -0.363), Digestion (-0.415) and for skeletal, muscle and connective $(-0.125)$ and central nervous system
(0.29). While for other systems leptokurtic types of distributions were observed as the value of kurtosis is more positive than normal distribution (kurtosis $=$ zero). Highest leptokurtic type distribution was recorded for endocrine gland, metabolism and nutrition followed by circulatory system (Fig. 1). The results of the K-S test are also presented in table 4. In this study as the calculated $\mathrm{D}$ value is less the standardized $\mathrm{P}$ 
Table 3. Total frequency class for each corporeal system and their respective species.

\begin{tabular}{lccccccccc}
\hline \multirow{2}{*}{ Corporeal systems } & \multicolumn{7}{c}{ Number of species in each frequency classes } \\
\cline { 2 - 8 } & $\mathbf{1}$ & $\mathbf{2}$ & $\mathbf{3}$ & $\mathbf{4}$ & $\mathbf{5}$ & $\mathbf{6}$ & $\mathbf{7}$ & $\mathbf{8}$ & $\mathbf{9}$ \\
\hline Respiration & 31 & 27 & 15 & 9 & 5 & - & - & - & - \\
Digestion & 33 & 21 & 19 & 13 & 16 & 7 & - & 2 & - \\
Reproduction & 44 & 18 & 6 & 2 & 1 & - & - & - & - \\
Fever & 46 & 13 & - & 4 & 1 & - & - & - & - \\
Blood and hematopoietic organ & 37 & 10 & - & 4 & 2 & - & - & - & - \\
Central nervous system & 33 & - & 10 & - & 5 & - & - & - & - \\
Genito-urinary system & 54 & - & 11 & - & 1 & - & - & - & - \\
Circulatory system & 16 & - & 2 & - & 1 & - & - & - & - \\
Sensory system & 28 & 7 & - & 3 & 1 & - & - & - & - \\
Endocrine gland, metabolism and nutrition & 21 & - & - & - & 1 & - & - & - & - \\
Skin and sub-cutanuous system & 32 & 26 & 14 & 11 & 3 & 1 & 1 & - & 1 \\
Skeletal, muscle and connective tissues & 37 & 28 & 26 & 11 & 7 & 5 & - & - \\
\hline
\end{tabular}

Table 4. Frequency distribution parameters for different corporeal systems.

\begin{tabular}{|c|c|c|c|c|}
\hline \multirow{2}{*}{ Corporeal Systems } & \multicolumn{2}{|c|}{$\begin{array}{c}\text { Normal distribution } \\
\text { parameters }\end{array}$} & \multicolumn{2}{|c|}{$\begin{array}{c}\text { Kolmogorov-Smirnov } \\
\text { (K-S test) }\end{array}$} \\
\hline & Skewness & Kurtosis & D Observed & Standardized P value \\
\hline Respiration & 0.785 & -0.363 & 0.231 & 2.15 \\
\hline Digestion & 0.652 & -0.415 & 0.182 & 1.925 \\
\hline Reproduction & 1.708 & 2.810 & 0.360 & 3.30 \\
\hline Fever & 1.801 & 2.77 & 0.429 & 3.420 \\
\hline Blood and hematopoietic organ & 1.709 & 2.09 & 0.413 & 3.0 \\
\hline Central nervous system & 1.29 & 0.29 & 0.418 & 2.89 \\
\hline Genito-urinary system & 2.017 & 3.29 & 0.492 & 3.99 \\
\hline Circulatory system & 2.25 & 4.09 & 0.495 & 2.15 \\
\hline Sensory system & 1.751 & 2.30 & 0.425 & 2.65 \\
\hline Endocrine gland, metabolism and nutrition & 4.07 & 15.26 & 0.539 & 2.52 \\
\hline Skin and sub-cutanuous system & 1.62 & 3.67 & 0.238 & 2.24 \\
\hline Skeletal, muscle and connective tissues & 0.822 & -0.125 & 0.197 & 2.09 \\
\hline
\end{tabular}

value, therefore we reject the null hypotheses $\left(\mathrm{H}_{0}\right)$ : the data follow the normal distribution and accept the alternative hypothesis $\left(\mathrm{H}_{\mathrm{A}}\right)$ that the data does not follow the normal distribution (Table 4).

A residual plot is a graph that shows the residuals on the vertical axis and the independent variable on the horizontal axis. If the points in a residual plot are randomly dispersed around the horizontal axis, a linear regression model is appropriate for the data; otherwise, a nonlinear model is more appropriate. In present study standard residuals analysis has indicated that the digestion (1.952) are the positive outliers while only two systems namely, fever (-1.15) and genito-urinary systems (-1.419) were the negative outliers (fig. 2). For digestion systems species like Achyranthies aspera, Aegle marmelos, Aloe vera, Cissus quadrangularis, Cynodon dactylon, Punica granatum, Tamarindus indica, Tephrosia purpurea and Vernonia cinerea made this system as outlier for their maximum uses as purgative, demulcent, anti-dysenteric, anodyne, laxative and use for ulcer, constipation and diarrhea. Out of 111 plant species only six species namely Cynodon dactylon, Fagonia indica, Murraya koenigii, Ocimum sanctum, Urginea indica and Vernonia cinerea are being reported as anti-vomiting.

For fever and genito-urinary systems, 101 different species have been reported for disease related to these two systems, this study revealed that out of 101 species only 30 species are common in both diseases related to these systems (Table 4). For fever 64 different plant species have been reported and out of which $92.18 \%$ (59) species were reported for one or two diseases related to this corporeal system, remaining 5 species (7.81) having potentiality to use for 3 to 4 different 
Respiration

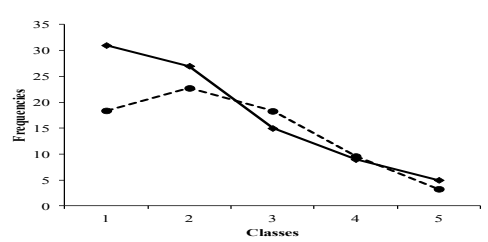

Digestion

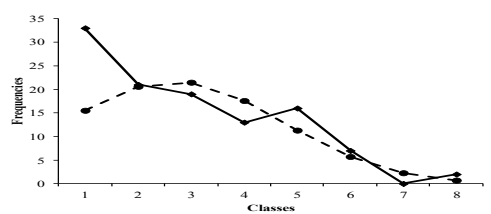

Reproduction

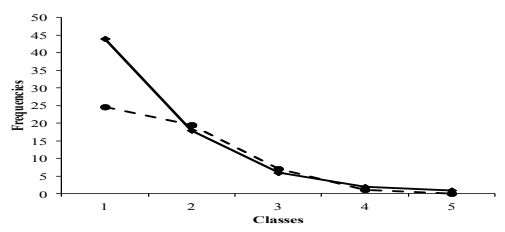

Fever

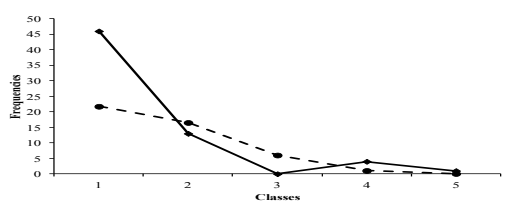

Blood

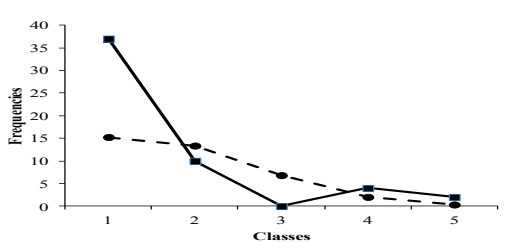

CNS

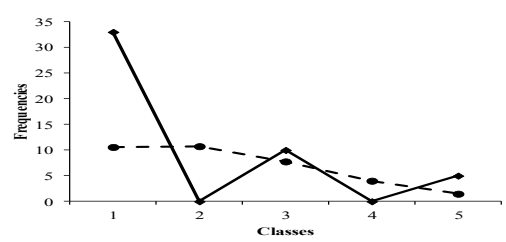

Genito

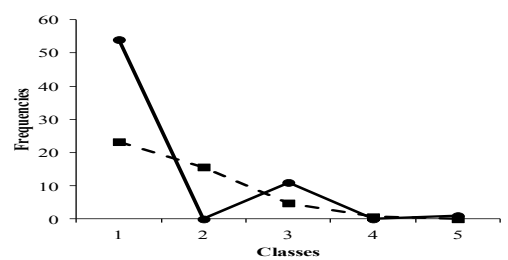

Circulatory

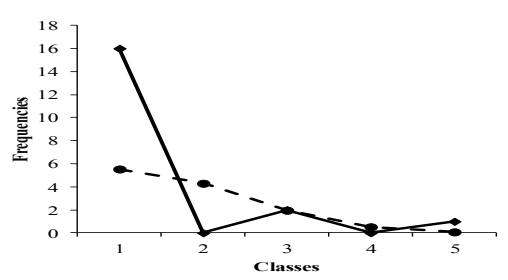

Sensory

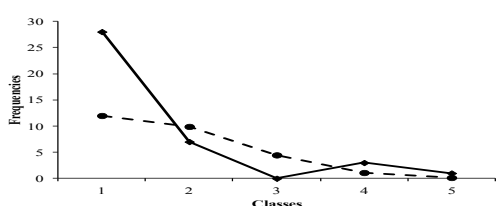

Endocrine

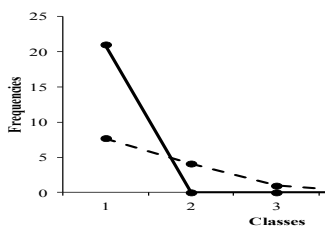

Skin

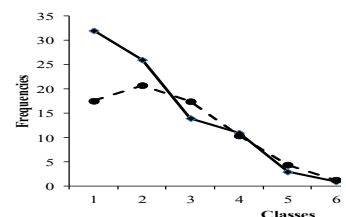

Skelatol

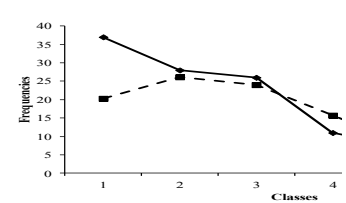

\section{$\leadsto$ Observed frequency $\quad-6$ Theoretical frequency}

Fig. 1. Observed and theoretical frequencies for various corporeal systems.

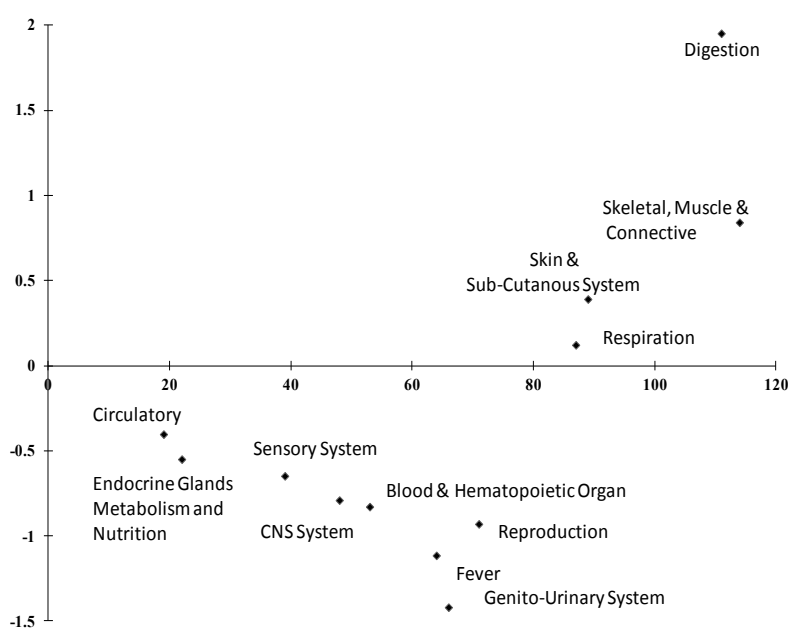

Fig. 2. Residual value analysis for corporeal systems.

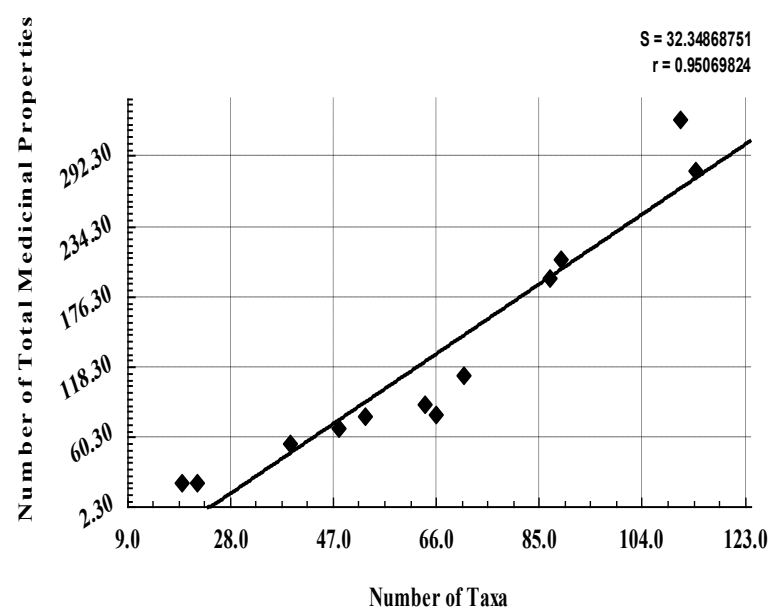

Fig. 3. Linear regression analysis between exploratory and dependent variables. 
diseases. These outlier's species are Azadirachta indica, Boerhavia diffusa, Cassia fistula, Phyllanthus fraternus and Tamarindus indica. These species have been reported for their properties to treat malaria, jaundice, and pneumonia and as antipyretic and anti-periodic. Similarly among 66 different species reported for diseases of genito urinary tract, $80 \%$ (53 species) are reported for only one disease related to this corporeal system, thus remaining species (13) are regarded as outliers for their ethno-medicinal properties related with more than one disease related with this system. Such outlier's species include Tribulus terrestris, Achyranthies aspera, Asparagus racemosus, Boerhavia diffusa, Catharanthus roseus, Cynodon dactylon, Pedalium murex, Phyllanthus fraternus, Tephrosia purpurea, Trianthema portulacastrum, Dalbergia sissoo and Solanum xanthcarpum. These species are being reported as diuretic and treatment for kidney stone and urinary infections. Thus for species like Cynodon dactylon, Fagonia indica, Murraya koenigii, Ocimum sanctum, Urginea indica and Vernonia cinerea clinical validations for their anti-vometing properties should be carried out, so that this scattered information can be promulgated scientifically. Similarly outlier's species related with fever and Genito-Urinary Systems like Azadirachta indica, Boerhavia diffusa, Cassia fistula, Phyllanthus fraternus, Tamarindus indica, Tribulus terrestris, Achyranthies aspera, Asparagus racemosus, Catharanthus roseus, Cynodon dactylon, Pedalium murex, Tephrosia purpurea, Tianthema portulacastrum, Dalbergia sissoo and Solanum xanthcarpum are pharmaceutical important because of their immense capacities to treat various diseases related with these two system.

In present study a linear regression equation has been established i.e. number of total medicinal properties $=-70.92+3.03 \times$ number of taxa $\left(R^{2}=0.95\right.$; $\mathrm{P}>0.05 \%)$ and it is graphically presented in fig. 3 . This linear relationships indicates that traditional ethno-medicinal knowledge for any species is linearly and positively associated with regional abundance/availability of species

\section{Conclusion}

Novelty of the present work is related with its quantitative parameters utilized for quantification of homogeneity for traditional medicinal knowledge. Other previous studies like Namasa et al., (2011) and Alsarhan et al., (2012) have utilized a informant consensus factor values (FIC) for quantification of community knowledge. However such previous studies did not document the direction of knowledge (homogeneity or heterogeneity). Rokaya et al., (2014) have showed the diversity and importance of medicinal plants used to treat gastro-intestinal disorders in the traditional health care system of Nepal. They have documented highest number of species for treatment of disease like diarrhea, stomach-ache and dysentery. The present top down approach for quantitative evaluation of homogeneity of ethnic-knowledge about arid and semi arid plants are very fruitful. These combined statistical approaches not only provide information about the wealth and patterns of ethnic knowledge, but it also provides an insight to know the level and strength of ethnic knowledge transmission, medicinal property of a species need to be clinically validated, identification of species that having high pharmaceutical values and relationships between abundance of a species and its ethnic knowledge. From this study, it's emerged that traditional ethno- knowledge particularly related with endocrine gland, metabolism and nutrition need to be documented and well quantified to avoid loss of traditional knowledge (as they are clumped in nature)

\section{REFERENCES}

Alsarhan, A., Sultana, N., Kadir, M.R.A. andAburjai, T. (2012). Ethnopharmacological survey of medicinal plants in Malaysia, the Kangkar Pulgai Region. International Journal of Pharmacology, 8 (8): 679-686.

Amiguet, V.T., Arnason, J.T., Maquin, P., Cal, V., Vindas, P.S. and Poveda, L. (2005). A consensus ethnobotany of the Q'eqchi' Maya of Southern Belize. Economic Botany, 59(1) 29-42.

Begossi, A. (1996). Use of ecological methods in ethnomedicine: Diversity indices. Economic Botany, 50: 280- 289.

Bennett, B.C. and Husby, C.E. (2008). Patterns of medicinal plant use: An examination of the Ecuadorian Shuar medicinal flora using contingency table and binomial analyses. Journal of Ethnopharmacology, 116(3): 422-430.

Bentley, R.A., Hahn, M.W. and Shennan, S.J. (2004). Random drift and culture change. Proceedings of the Royal Society B-Biological Sciences, 271(1547): 1443-1450.

Brower, J.E. and Zar, J.H. (1977). Field and Laboratory Methods for General Ecology. Dubuque, Iowa: WC Brown Publishers

Di Cosmo, N. (2002). Ancient China and its enemies: The rise of nomadic power in East Asian history. Cambridge, UK: Cambridge University Press.

Henrich, J. and Henrich, N. (2010). The evolution of cultural adaptations: Fijian food taboos protect against dangerous marine toxins. Proceedings of the Royal Society B, 277: 3715-3724.

Khan, S.M., Harper, D., Page, S. and Ahmad, H. (2011). Residual value analysis of the medicinal flora of the western Himalayas: The naran valley, Pakistan, Pakistan Journal of Botany, 43: 97-104.

Krebs, C.J. (1999). Ecological Methodology. NY: An Imprint of Wesley Longman, Inc., P. 620.

Kumar, S., Mathur, M., Kushwaha, N., Goyal, S., Chauhan, A and Parween, F. (2014). New traditional herbls from India arid zone for curing rheumatism. Asian Agri-History 18 (2): 133-144.

Leonti, M. (2011). The future is written: Impact of scripts on the cognition, selection, knowledge and transmission of medicinal plant use and its implications for ethnobotany and ethnopharmacology. Journal of Ethnopharmacology 134: 542-555.

Ludwig, J.A. and Reynold, J.F. (1988). Statistical Ecology-A Primer on Method and Computing. Toronto: John Wiley and Sons.

Mathur, M. (2014b). Ethnic-Knowledge: Documentation and 
their Interpretation. Agrobios Publication, Jodhpur, India. P. 344.

Mathur, M. (2005). Ecology and prospecting of some medicinal plants of aphrodisiac potential. $\mathrm{PhD}$ thesis, Department of Botany, Jai Narayan Vyas University, Jodhpur, India.

Mathur, M. (2012). Use of quantitative ethnobotany for assessing potential and conservation priorities of the Indian Thar desert medicinal plants claimed for central nervous disorders. Medicinal Plants: International Journal of Phytomedicine and Related Industries, 4 (3): 143-153.

Mathur, M. (2012a). Complementary and alternative medicines use for gastro-intestinal disorders, International Journal of Pharma Research and Review, 1 (7): 16-36.

Mathur, M. (2012b). Use of quantitative ethno botany for assessing potential and conservation priorities of the Indian Thar Desert medicinal plants claimed for central nervous disorders. Medicinal Plant International Journal of Phytomedicine and Related Industry, 4: 143-153.

Mathur, M. (2013c). Evaluation of conservation priorities of arid plants by using approaches of quantitative ethnobotany and ecosystem services. The Journal of Ethnobiology and Traditional Medicine 120: 608-619.

Mathur, M. (2013d). Autocorrelation simulation studies for horizontal transmission of ethno-medicinal- knowledge related with two corporeal systems. International Research Journal of Biological Sciences, 2 (12): 18-29.

Mathur, M. (2014a). Attributes of Plant Spatial Analysis. Today and Tomorrow Printer and Publishers, New Delhi, India. P. 365

Mathur, M. and Sundaramoorthy, S. (2013a). Census of approaches in used in quantitative ethnobotany. Studies in Ethnomedicine 7 (1): 31-58.

Mathur. M. and Sundaramoorthy, S. (2013b). Economic assessment and conservation priorities of the Indian Thar desert medicinal plants. Indian Journal of Natural Products and Resources 4 (3): 283-294.

Meeeiros, M.F.T., de Silva, P.S. and de Albuquerque, U.P. (2001). Quantification in Ethnobotanical research: an overview of indices used from 1995 to 2009. Sitientibus serie Ciencias Biologicas, 11 (2): 211-230

Moerman, D.E. (1996). An analysis of the food plants and drug plants of native North America. Journal of Ethnopharmacology, 52: 1-22.

Namsa, N.D., Mandal, M., Tangjang, S. and Mandal, S.C. 2011. Ethnobotany of the Monpa ethnic group at Arunachal Pradesh, India. Journal of Ethnobiology and Ethnomedicine, 7 (31): 1-14

Payne, L.X., Daniel, E.S., Parrish, J.K. and Temple, S.A. (2005). Quantifying spatial pattern with evenness indices. Ecological Applications, 15 (2): 507-520.

Rokaya, M.B., Uprety, Y., Poudel, R.C., Timsina, B., Munzbergova, Z., Asselin, H., Tiwari, A., Shrestha, S.S. and Sigdel, S.R. (2014). Traditional uses of medicinal plants in gastrointestinal disorders in Nepal. Journal of Ethnopharmacology, 158, 221-229.

Signorinei, M.A., Piredda, M., Bruschi, P. (2009). Plants and traditional knowledge: An ethno-botanical in-vestigation on Monte Ortoben Nuro, Sardinia. Journal of Ethnobiology and Ethnomedicines, 5: 6-20.

Tehrani, J.J., and Collard, M. (2009). On the relationship between inter-individual cultural transmission and population-level cultural diversity: A case study of weaving in Iranian tribal populations. Evolution and Human Behavior, 30: 286-300.

Tuomisto, H. (2012). An updated consumer's guide to evenness and related indices. Oikos, 121: 1203-1218.

Wilcox, R.R. (2001). Fundamental of Modern Statistical Method (Springer, New York).

Williams, V.L., Witkowski, E.T.F. and Balkwill, K. (2005). Application of diversity indices to appraise plant availability in the traditional medicinal markets of Johannesburg, South Africa. Biodiversity and Conservation, 14: 2971-3001.

Yang, S.Z. and Gao, Y.J. (2011). A preliminary study on diverse plant uses of Rukai tribe in wutai district of pingtung county, Southern Taiwan. Taiwania, 56: 7-16. Zar, J. H. (1984). Bio-Statistical Analysis. Englewood Cliffs: Prentice-Hall, Inc. 